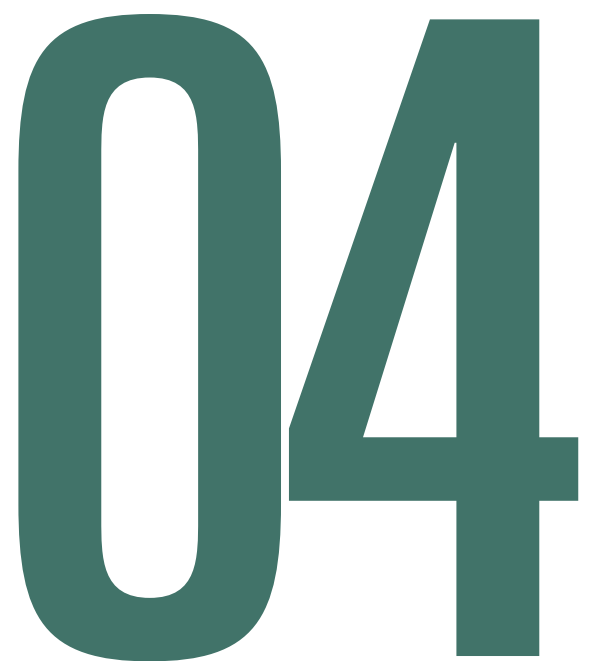

\title{
WORDPRESS, LA EVOLUCIÓN DE LA PÁGINA WEB.
}

Oscar Edgar Palafox Flores Universidad de Sonora oscar_palafox@hotmail.com Maestro en Competitividad Organizacional, Profesor en el Departamento de Arquitectura y Diseño Gráfico de la Universidad de Sonora

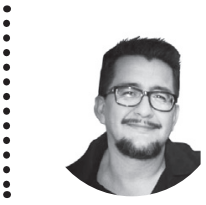

MADGU. Mundo, Arquitectura, Diseño Gráfico y Urbanismo ISSN: en tramite 


\title{
Resumen Artículo
}

Palabras claves: Web, Redes sociales, Trending Topic, Like, News Feed, Smartphones, Wordpress. Key words:

\begin{abstract}
Resumen
La Word Wide Web avanza a gran velocidad junto a las tecnologías relacionadas con el Internet. Las redes sociales son la plataforma donde se realiza el porcentaje más alto de acciones dentro del ciberespacio; por ello, conocerlas, entenderlas y aprovechar sus ventajas, hacen la diferencia en la mercadotecnia y branding actual. Los productos, servicios y demás ofertas, anteriormente estaban diseñados para que el usuario se adaptara a ellos; ahora, estos proveedores, entre ellos los digitales, están obligados a rediseñarse en función de lo que las personas quieren.

En este artículo se habla de Wordpress, considerada la evolución de las tradicionales páginas de internet.

Abstract

The World Wide Web is advancing at great speed along with Internet-related technologies. Social networks are the platform where the highest percentage of actions is carried out within cyberspace; therefore, knowing them, understanding them and taking advantage of their advantages make the difference in current marketing and branding.

The products, services and other offers previously were designed for the user to adapt to them; now, these providers, including digital ones, are forced to redesign themselves based on what people want.

In this article we will talk about WordPress, considered the evolution of traditional internet pages.
\end{abstract}


Oscar Edgar Palafox Flores

\section{Marco referencial}

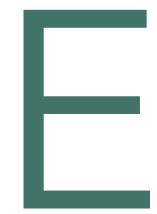

n la actualidad nos encontramos con diversas redes sociales dominando el internet de manera apabullante; como referencia, bandas musicales que tienen sus inicios y base de fanáticos obtenido de redes sociales musicales tales como Soundcloud, por mencionar una de las más populares, los lanzan al estrellato y gracias al compartir que los usuarios otorgan, cada banda va ascendiendo en las listas de popularidad en el mundo, lo que los ubica en posición para obtener contratos con disqueras y distribuidoras a nivel internacional.

Desde lo más trágico hasta lo más gracioso puede convertirse en un Trending Topic, y aunque son literalmente quince minutos de fama, es el gusto de los usuarios, lo que las grandes corporaciones buscan para llegar a ellos. Es aquí donde plataformas como Wordpress aprenden de estos News Feed de red social, tomando los elementos esenciales que las hacen tan populares entre los usuarios, para hacer uso de sus ventajas competitivas como lo son su formato, maquetación, estilo y lenguaje con el que se dirigen a los usuarios.

El inicio de esta tendencia que sigue expandiéndose, creando el despunte de Wordpress, fueron los Blogs, páginas que cualquier persona puede crear, administrar y sobre todo enriquecer de información a su gusto. Son estos foros de opinión los que catapultaron a Wordpress como la nueva faceta de las páginas de internet y que abrieron aún más el internet a todos los usuarios. 


\section{Análisis}

Podemos iniciar preguntando ¿son las páginas web algo del pasado?, a lo que podríamos señalar que son los Smartphones o comúnmente llamados celulares, los que se han encargado de encasillarlas como algo histórico. El diseño de páginas web, ha pasado a un segundo plano, siendo desplazados por las redes sociales y las aplicaciones móviles, los cuales son la herramienta número uno en el mundo para dar a conocer servicios y productos, así como crear nuevas necesidades en la sociedad.

Son los dispositivos móviles, cuyos navegadores utilizados para visitar páginas web no han adaptado su visualización y acceso a través de los años, los responsables de convertir su uso en una experiencia desagradable.

Las redes sociales como Facebook, Instagram y Twitter, entre muchas más, son multitasking; cada vez son más versátiles y además su funcionamiento se basa en la practicidad, eficiencia, diseño minimalista y una interfaz totalmente intuitiva, siendo su mayor ventaja la interactividad de poder conectarte con un representante de la marca en vivo de manera inmediata, en sus diferentes redes sociales.

Los desktops u ordenadores de escritorio, son la plataforma para la que se diseñaron inicialmente las páginas de internet. En la actualidad, en los hogares de la familia promedio, estos dispositivos dieron paso a las tabletas y al Smartphone. Este último está diseñado para quienes utilizan esencialmente redes sociales y múltiples aplicaciones. Por su parte, el mercado de los ordenadores de escritorio y laptops, han pasado a ubicarse en un mercado para profesionistas, empresas, industrias y especialistas; esta evolución tecnológica no significa que estos desaparecerán próximamente.

Wordpress representa una opción de página web, con características emuladoras de una red social, dando con ésta, una ventaja competitiva sobre las páginas de internet tradicionales, además de que los sitios en Wordpress, son compatibles con las diferentes plataformas de red social del mercado, logrando una sinergia sin precedentes. 
Con el propósito de dar a conocer las estadísticas de las preferencias y tenencias de los usuarios de la red en México, existe la Asociación de Internet.mx, la cual realiza anual y eventualmente estudios sobre los hábitos de los internautas mexicanos, redes sociales, comercio electrónico, banca en línea, protección de datos personales, búsqueda de empleo e infraestructura, entre otros.

En su 13avo. Estudio Sobre Los Hábitos de los Usuarios de Internet en México, la Asociación de Internet.mx (2017) concluye que habría más de 70 Millones de internautas en México para 2017, que significa el $63 \%$ de penetración entre la población. El 52\% de los internautas en México se encuentra conectado las 24 horas. El tiempo promedio de conexión en internet es de 8 horas con 1 minuto, lo que es 47 minutos más que en 2016.

El mexicano prefiere conectarse mediante un dispositivo móvil en el hogar o contratando un plan de datos. Nueve de cada 10 internautas poseen Laptops y Smartphone, con lo que el uso de la PC de escritorio disminuye dramáticamente, creciendo el uso de tabletas.

Las redes sociales permanecen como la principal actividad en línea; ganan terreno actividades como mailing y búsqueda de información. Confirmando este cambio de hábitos de consumo por parte de los usuarios, las compras repuntan, así como las finanzas en línea.

Es importante destacar que el segmento joven, empuja estos cambios de hábitos en la población mediante la adopción de tendencias; por ello, Facebook se mantiene como la primera red social en México. Igualmente se experimenta un gran crecimiento de Instagram, Linkedln y Snapchat, debido al también incremento en el uso de redes sociales respecto a 2016, (+7).

Los usuarios pasan conectados en internet o en alguna red social el 38\% de su tiempo; Smartphone es el principal dispositivo para acceder a alguna red social. Ocho de cada 10 internautas utilizan estos dispositivos. 
El uso de Smartphone en redes sociales, ha impulsado el crecimiento de usuarios, en específico con redes que nacieron sólo en versión móvil, como Snapchat, WhatsApp e Instagram. El uso de PC y Laptop se ha enfocado a redes sociales alternas o específicas como Slideshare, Linkedln y Flickr, que por su naturaleza facilita su uso en estos dispositivos.

Basándonos en la información que señala que un gran porcentaje del público en México utiliza las redes sociales para interactuar con el resto del mundo, así como datos sobre el mercado y sus comportamientos de compra, ello nos hace reflexionar en las palabras de Correa (2013), quien señala que vivimos en una sociedad pendiente de lo último en tendencias, modas y estilos, en el que todo es efímero y desechable.

Con respecto al diseño, podemos argumentar que el verdadero diseño permanece al margen de ello, no está pendiente de manías superficiales, ni mucho menos de modas que harán que él se vea anticuado o desencajado, dentro de un par de años o en el futuro. El buen diseño evita ir con la moda buscando más la funcionalidad.

El dispositivo número uno se encuentra en la palma de la mano de las personas a las que queremos llegar, su teléfono celular, el cual se utiliza principalmente para el manejo de Apps, en menor medida páginas web y por extraño que parezca, para hacer llamadas telefónicas, que es para lo último que se utiliza; así que no es ningún secreto que en los últimos años, la tendencia al uso de páginas haya disminuido; pero aquí es donde entran formatos de página web, como lo es Wordpress (2017) como un sistema de gestión de contenidos o CMS (por sus siglas en inglés, Content Management System), enfocado a la creación de cualquier tipo de página web.

Originalmente alcanzó una gran popularidad en la creación de blogs, para convertirse con el tiempo en una de las principales herramientas para la creación de páginas web comerciales, transformándose en una de las más importantes herramientas para el diseño web por ser un software libre. 
Considerar Wordpress en la nueva era de las páginas web, desde su lanzamiento el 26 de mayo de 2003, obedece simple y sencillamente a su maquetación; es decir, la esencia de su diseño, se ha ido perfeccionando en un hibrido de un blog y una red social, siendo este último el diseño preferido por los usuarios en la actualidad. Las personas están tan familiarizados con la interfaz y algoritmos de las redes sociales, que al visitar cualquier página web, creada en Wordpress, intuitivamente conocen su funcionamiento estilo línea de tiempo; incluso cuando es la primera vez que visiten el sitio, el usuario suele desenvolverse con gran facilidad dentro de él, lo que puede garantizar que la persona volverá a visitarla.

Estableciendo su diseño basado en red social, la página de cualquier proyecto a futuro debe adecuar su contenido a este estilo, lejos y atrás, quedan atrás una serie de aspectos que contenían los sitios; la velocidad de navegación, así como el corto tiempo de atención que se tiene de los espectadores, debe ofrecerles lo que buscan: notas cortas con información actual, es decir actualizaciones diarias.

Lo más buscado en los sitios web por las personas, en cualquier situación, viene a ser información como ubicación de la tienda, sucursales, productos disponibles, horarios, precio, contacto y servicios que ofrecen. Dicha información debe estar clara y oportunamente dentro del sitio; buscando la máxima funcionalidad, debe hacerse un diseño minimalista. Este, no debe ser basado en una moda, sino todo lo contrario, es una forma de hacer las cosas; todo el esfuerzo se enfoca en lo importante, no utiliza detalles ni ornamentos adicionales, sin embargo, lo que sí se puede considerar como de tendencia es el minimalismo, el cual tiene una belleza natural que se traduce en la simplicidad de las cosas y su funcionalidad natural.

El contenido debe ser claro, directo y con poca saturación de color; al contrario, lo recomendable es usar paletas sencillas de alto contraste y legibilidad. Siempre hay que recordar que se diseña para visualización en pantalla. Es en este diseño donde aplica la frase entre menos palabras mayor contundencia del mensaje. 
En una web saturada, los usuarios tienden a evitar los diseños cargados de elementos que inclusive hacen más lento el dispositivo, lo que además alarga el tiempo de espera de carga. La famosa frase de Loading, debe evitarse lo más posible, pues la reacción de cualquier usuario ante esta señal, es salir del sitio web.

\section{Conclusión}

Definitivamente la actualidad del diseño web es adaptarse al usuario, a sus hábitos y preferencias.

Clave en el éxito del diseño para entornos digitales, es el seguir a nuestros usuarios y conocer sus gustos, horarios claves de su presencia en línea, aplicaciones que más utiliza, las redes sociales en las cuales comparte su vida con familiares y amigos, entre otras, para así poder presentarle de la mejor manera el producto o servicio que buscamos ofrecerle.

Los usuarios evitan la publicidad tradicional; con los conocimientos que tenemos de sus gustos musicales, comerciales de alimentación y todo lo demás que nos dejan conocer a través de sus cuentas personales, debemos sugerir que el estilo del servicio que ofrecemos sea compatible al suyo o mejor aún ir un paso adelante, mostrándole lo que desearán en su vida y hacerle ver que realmente somos la mejor opción para ellos. Wordpress es una excelente herramienta para lograr este objetivo, debido a su evolución dentro del diseño de páginas que trabajan en conjunto con las redes sociales de mayor demanda.

\section{Referencias}

Correa L. (2013). Diseño gráfico y web para la posteridad. FOROALFA. Recuperado de https://foroalfa.org/articulos/diseno-grafico-y-web-para-la-posteridad

Asociación de Internet.mx. (2017). 13avo Estudio Sobre Los Hábitos de los Usuarios de Internet en México. Asociación de Internet.mx. Recuperado de https://www.asociaciondeinternet.mx/es/component/remository/Habitos-de-Internet/13-Estudio-sobre-los-Habitos-de-los-Usuarios-de-Internet-en-Mexico-2017/lang,es-es/?Itemid=

Wordpress. (2017). El 29\% de Internet utiliza WordPress. Wordpress. Recuperado de https:// es.wordpress.com

MADGU. Mundo, Arquitectura, Diseño Gráfico y Urbanismo 